\title{
Bibliometric Analysis of Knowledge Networks and Creativity
}

\author{
Dongxia Huo ${ }^{1} \&$ Sarana Photchanachan ${ }^{2}$ \\ ${ }^{1} \mathrm{PhD}$ Candidate, Management, School of Management, Shinawatra University, Thailand \\ ${ }^{2}$ School of Management, Shinawatra University, Thailand \\ Correspondence: Dongxia Huo, 99 Moo 10 Bang Toey, Sam Khok District, Pathum Thani Thailand. E-mail: \\ huodx@139.com
}

Received: February 3, 2021

Accepted: March 26, 2021

Online Published: April 2, 2021

doi:10.5539/ijbm.v16n5p35

URL: https://doi.org/10.5539/ijbm.v16n5p35

\begin{abstract}
Despite the sharp increase in awareness of workplace knowledge networks and creativity research, no attention has been paid to objectively visualizing the evolution of this fast-growing area to complement prior qualitative reviews. This bibliometric analysis involves an examination of 341 global knowledge networks and creativity articles in management-related research in the Web of Science database. Using CiteSpace V visualization literature measurement software, the knowledge map of the knowledge networks and creativity research was drawn using the scientific metrology knowledge graph research method. We conduct Publication time analysis, country/region analysis, journal co-citation analysis, author co-citation, document co-citation, the time-zone visualization analysis on research literature in the field of knowledge networks and creativity, the major researchers and topics in the field of knowledge network, and creativity are clearly presented. Moreover, we summarize the developing trends of knowledge networks and creativity research. On this basis, our review demonstrates the systematic development of literature and identifies trends to advance knowledge networks and creativity research.
\end{abstract}

Keywords: bibliometric review, knowledge network, creativity, visual analysis

\section{Introduction}

In the 1950s, since the concept of "creativity" was put forward, it quickly set off a wave of creativity research in academia (Bavelas \& Alex, 1950). After 70 years of development, it is still widely concerned by scholars until today. The impact of social networks and knowledge structure on creativity is of great significance. It has become one of the most important research topics in the field of innovation management. In this paper, the knowledge networks refer to the network formed by the knowledge subject (individual, team or organization, etc.) as the network node and the information or knowledge flow as the connection, including social network, co-author network, problem-solving network, consulting network, joint venture network, organizational alliance and other forms. The knowledge subject involves the characteristics of the knowledge subject, including the depth and breadth of the knowledge subject. Creativity refers to the ability to generate the novel and useful ideas. Creativity cannot be separated from a certain knowledge environment, and knowledge networks can provide heterogeneous or tacit knowledge to the knowledge subject, thus promoting creativity. In the context of open innovation, the research on the relationship between knowledge networks and creativity is booming in the academic circle. Scholars have found that the characteristics of knowledge, the structure, relations, and node characteristics of knowledge networks affect direct and indirectly creativity through knowledge transfer, dissemination or diffusion (Phelps, Heidl, \& Wadhwa, 2012; Perry-Smith, 2006; Zhou, Shin, Brass, Choi, \& Zhang, 2009). At present, relevant empirical research conclusions are scattered in the fields of technological innovation, management, and psychology at different levels of research, it is necessary to carry out systematic integration analysis.

\section{Method and Data Collection}

\subsection{Method}

The purpose of our research is to provide a visual impression of knowledge networks and creativity and represent their evolution from 1996 to 2020 by using bibliometric mapping (Chen, 2006). This paper adopts the knowledge graph in scientific metrics to visually analyze the relevant literature on the knowledge networks and creativity in the international journal, while the concept of the scientific knowledge graph first appeared in a 
seminar organized by the National Academy of Sciences in 2003 (Chen, Liu, Chen, \& Hou, 2008). The progress of computer technology has brought more tools to draw the scientific knowledge graph, especially CiteSpace knowledge visualization tools have been widely recognized. CiteSpace can display the evolution of the knowledge field on a citation network map, and also automatically identify the research frontiers represented by the citation node literature and clusters as the knowledge base (Chen, Chen, Liu, Hu, \& Wang, 2015). CiteSpace also can be used to construct and display the bibliometric map based on the differences in distance, size, and density between nodes, which can be used in the cluster view, overlay view and density view to evaluate the research direction and hot spots of the literature (Chen, 2013). Through combining with the advantages of the visualization methods, this paper uses CiteSpace $\mathrm{V}$ to draw a knowledge networks and creativity research knowledge map and analyzes the research status taking the national, institutional, and literature citations as nodes. In addition, draw the co-occurrence and clustering knowledge map of keywords, in order to better seek the knowledge networks and creativity research evolution and trends.

\subsection{Data Collection}

In our research, we review the knowledge networks and creativity literature for the period 1995-2020 from the Web of Science database. The data pool takes the Web of Science database (including SCI-EXPANDED, SSCI, A\&HCI, CPCI-S, CPCI-SSH, ESCI, CCR-EXPANDED) as the data source for the subject retrieval, and this research defines the searched keywords to cover all the research literature of knowledge networks and creativity research. The search formula is TS $=$ ((social network) or (Knowledge Depth) or (Knowledge Breadth) or (knowledge network) or (knowledge management)) and TS = (creativity). This research limits the time to 1995-2020, covering papers in all disciplines and represented in the English language. Finally, we obtain full bibliographic records of 519 articles, which are cited 24,251 times up to January 8, 2021.

\section{Bibliometric Analyses and Results}

\subsection{Publication Time Distribution}

The publication time and annual distribution of knowledge networks and creativity are shown in Figure 1. The first paper in this field was published in 1996. From 1996 to 2005, the number of published papers was relatively small, less than 10 per year. It began to grow slowly in 2006 and exceeded 35 per year after 2012, showing a booming trend. It can be seen that the number of articles issued by the knowledge networks and creativity is on the rise year by year, especially after 2012, which has become a hot spot of management research. With the rapid development of science and technology, the development of mobile Internet, and big data, the research on knowledge networks and creativity will still be the focus of future research.

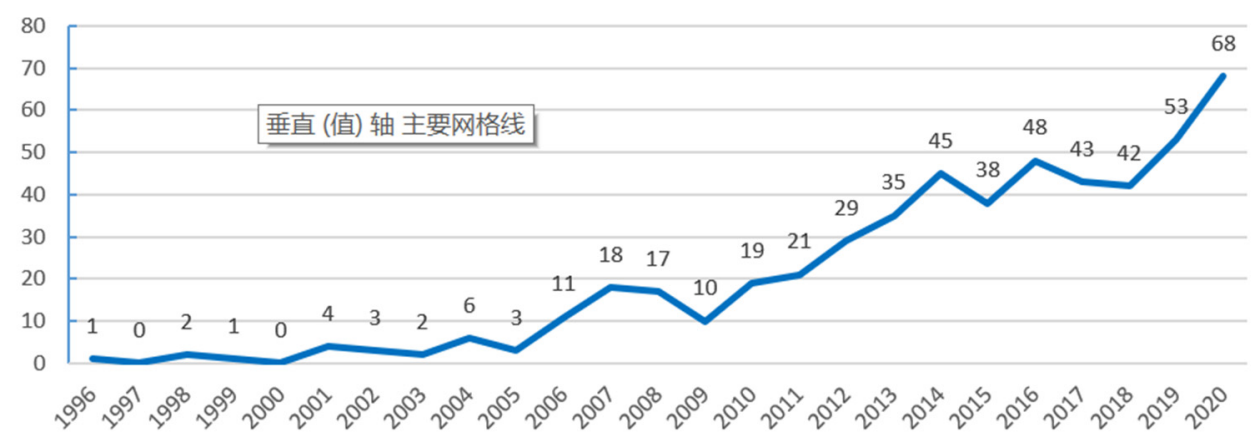

Figure 1. Publication time distribution of knowledge networks and creativity research

\subsection{Distribution of Countries/Regions}

According to 519 records on knowledge networks and creativity in the data pool, the research obtains national/regional distribution knowledge networks graph by using CiteSpace V software. The size and color of annual ring can visually display the number and center degree of the papers, indicating the larger annual ring can bring more published papers, and the darker annual ring can bring the stronger centrality. After obtaining the national/regional knowledge graph on knowledge networks and creativity (Figure 2), we can acquire 65 nodes, 125 connections and the network density of 0.073 . In detail, figure 2 shows the research on creativity has focused on three countries: USA, China and England. The contribution rate of USA literature can be the largest (151 papers) from the publishing frequency of papers in each node. It can demonstrate that USA is more active in the knowledge 
networks and creativity research field, followed by China (81), England (58), Netherlands (35), Italy (34), Spain (33), Australia (28), France (26), Germany (24), and Canada (19). USA has the largest centrality from the perspective of each node (seen in Table 1), indicating its obvious intermediary role in the research process. There are many studies conducted through this node, but its impact on the network structure can be the largest. The centrality of China is 0.19 followed by countries like USA and England, so it is still necessary to further strengthen the cooperation with other countries.

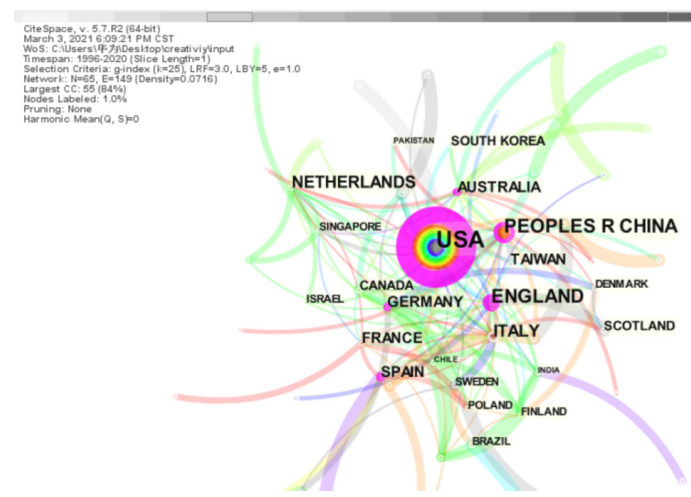

Figure 2. Countries/regions co-occurrence network of knowledge networks and creativity research

Table 1. Distribution table of countries/regions

\begin{tabular}{llll}
\hline No. & Countries & Frequency & Centrality \\
\hline 1 & USA & 151 & 0.62 \\
2 & CHINA & 81 & 0.19 \\
3 & ENGLAND & 58 & 0.22 \\
4 & NETHERLANDS & 35 & 0.07 \\
5 & ITALY & 34 & 0.02 \\
6 & SPAIN & 33 & 0.13 \\
7 & AUSTRALIA & 28 & 0.18 \\
8 & FRANCE & 26 & 0.07 \\
9 & GERMANY & 24 & 0.11 \\
10 & CANADA & 19 & 0.002 \\
\hline
\end{tabular}

\subsection{Journal Co-Citation Analysis}

Journal co-citation analysis can indicate connections among journals and represent the distribution of the existing knowledge in the knowledge networks and creativity domain (Tsai \& Wu, 2010). Figure 3 displays the network of the most co-cited journals that have published knowledge networks and creativity-related articles. It is worth noting that the top five influential journals are all top-tier management journals (i.e., Academy of Management Journal, Organization Science, Academy of Management Review, Administrative Science Quarterly, Journal of Applied Psychology), indicating that knowledge networks and creativity is a popular topic attracting significant attention from researchers in previous three decades.

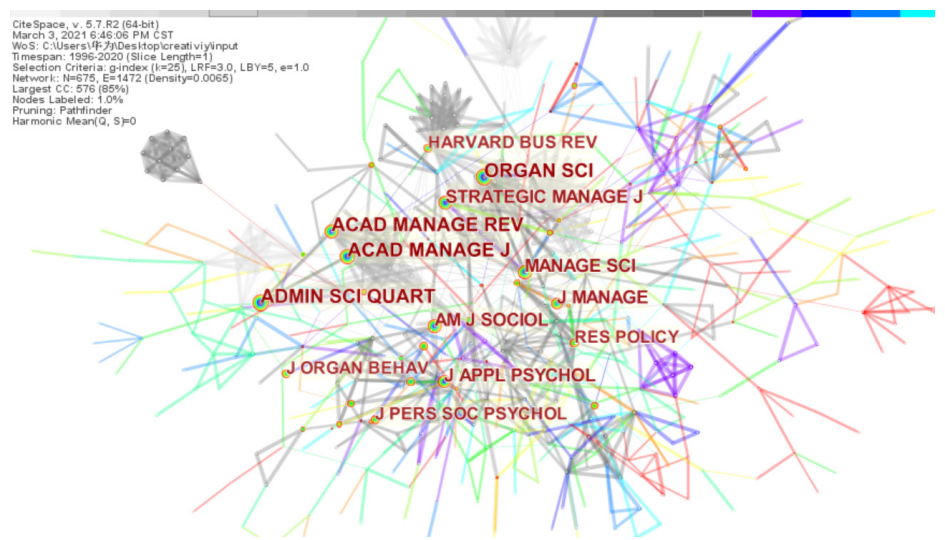

Figure 3. Journal co-citation network of knowledge networks and creativity research 


\subsection{Author Co-Citation Analysis}

By using author co-citation analysis, we can reveal the authors' contribution to intellectual advancement in knowledge networks and creativity research. The co-cited relationship of authors can be demonstrated in an author co-citation network, which is calculated based on the frequency of co-citation in primary documents (Liu \& Chen, 2012). In order to highlight the influential authors who have made a significant impact on knowledge networks and creativity research, we only label the authors with high co-cited frequency. Based on the articles published between 1996 and 2020, the visualization of the author co-citation network is displayed in Figure 4. In the author co-citation network, nodes and edges stand for each author and co-citation relationship, respectively. The size of every node is consistent with the frequency of author co-citation (Antonakis, Bastardoz, Jacquart \& Shamir, 2016). Five nodes stand out clearly as landmark authors: Perry-Smith JE, Amabile TM, Burt RS, Nonaka I, Hansen MT. We can speculate that these five authors play an important role in the evolution of knowledge networks and creativity research. In the author co-citation network, the pivot nodes, which stand for a turning point with two groups of communities holding different viewpoints, are highlighted with a purple ring. The pivot nodes (i.e., Perry-Smith JE, Amabile TM) also make key contributions during the evolution process.

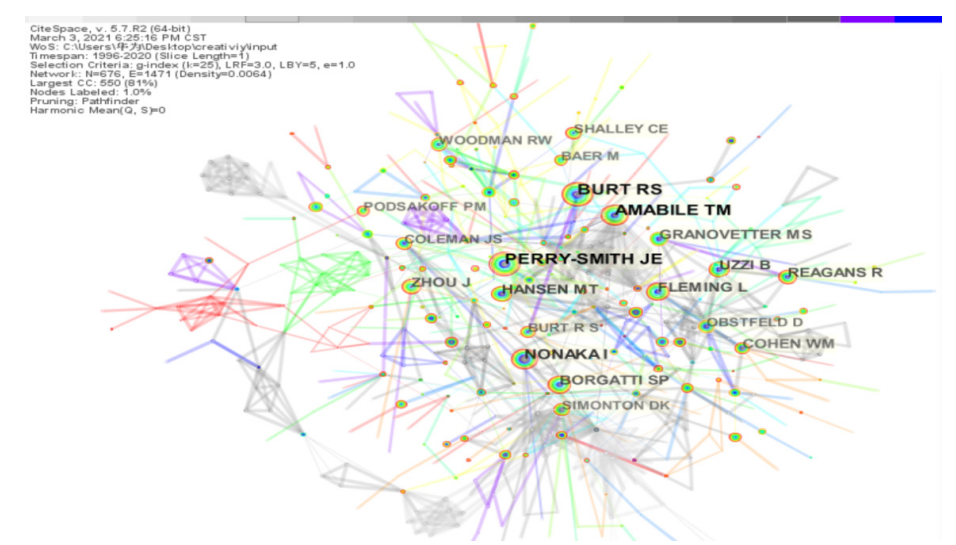

Figure 4. Author co-citation network of knowledge networks and creativity research

\subsection{Document Co-Citation Analysis}

The primary objective of this section is to reveal the landscape and paradigm development of knowledge networks and creativity research by conducting document co-citation analysis (Liu et al., 2015). Highly cited literature can be called basic knowledge in bibliometrics. As the classic in this field, they can reflect the overall knowledge foundation of the field. Through analyzing the highly cited literature, this paper attempts to reflect the basic knowledge of knowledge networks and creativity. When one particular article cites both reference $\mathrm{A}$ and $\mathrm{B}$, then reference $\mathrm{A}$ and $\mathrm{B}$ are defined as a co-citation linkage. We display the documents with high co-citation frequency to highlight the landmark works. The document co-citation network of knowledge networks and creativity literature (1996-2020) is presented in Figure 2 and Table 2. Five articles are identified as the most co-cited: Perry-Smith JE (2006), Zhou J (2009), Fleming L (2007), Baer M (2010), Phelps C (2012). These five articles are the most influential knowledge networks and creativity works, providing new and comprehensive insights into knowledge networks and creativity research and should be read by newcomers. For example, Perry-Smith JE (2006) explores the direct and interactive effects of relationship strength, network position, and external ties on individual creative contributions. Zhou J, et al. (2009) examined the influence of social networks and conformity value on employees' creativity. 


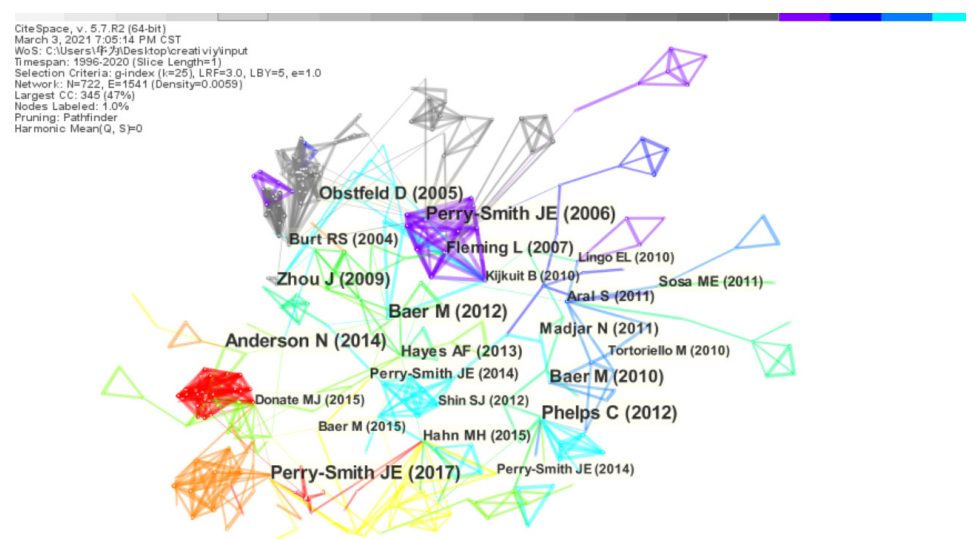

Figure 5. Document co-citation network of knowledge networks and creativity research

Table 2. Distribution table of countries/regions

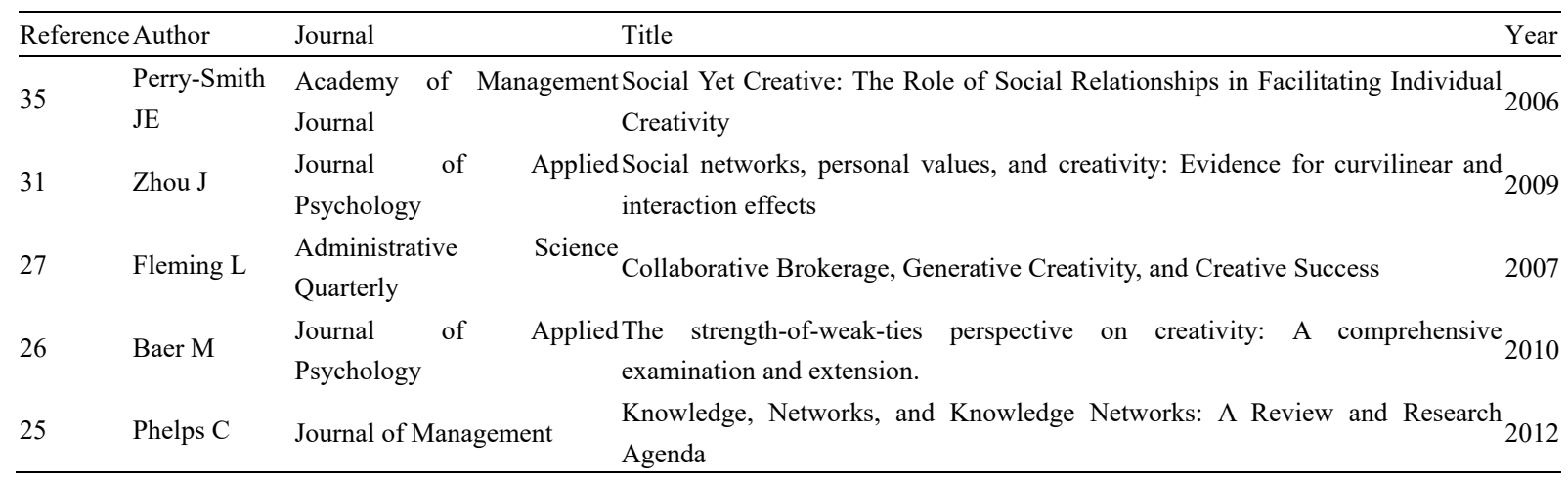

\subsection{The Time-Zone Visualization Analysis}

Keywords are the core of the paper; they are the subject of a high degree of synthesis and conciseness. keyword co-occurrence analysis is conducted to show the evolving research frontiers of knowledge networks and creativity concepts over the period time from 1996 to 2020. As indicated in Figure 4, the time-zone visualized graph represents the evolution of Knowledge networks and creativity concepts from left to right by arraying vertical strips each standing for a time-slice (Chen, 2006). In the keyword co-occurrence network, the node represents each knowledge networks and creativity theme and the size of each node represents the frequency of keyword co-occurrence (Zhu et al., 2018).

In the first period (1996-2001), the keywords that appear are knowledge management, creativity, product design, innovation integration, e-learning, knowledge capture, and new learning, which is commonly studied in knowledge management research, is the most concerning theme in knowledge management and creativity research.

In the second period (2002-2008), social network starts to emerge as a new theme. The keywords that appear are creativity, information retrieval, knowledge management, model, information, information management, design, absorptive capability, perspective, expected evaluation, social network, product development, capability and diversity. The research on the structural characteristics of knowledge networks and creativity has begun to rise. In the same period, there have also been many studies concentrating on knowledge management and performance.

In the third period (2009-2014), the keywords that appear are innovation, communication, management, perspective, employee creativity, behavior, organization, collaboration, transformational leadership, community, weak tie, mediating role, social network and knowledge management. The field of organizational behavior and human resources pay more attention to the research on this topic. At the same time, the subject characteristics and creativity in the knowledge networks are more concerned.

In the last period (2015-2020), the keywords that appear are organization, absorptive capacity, product development, culture, consequence, narcissism, innovation behavior, social network site, gender, attitude, intrinsic motivation, social network analysis, structural hole, knowledge sharing, leadership, orientation, 
entrepreneurship and climate. More and more attention has been paid to the relationship between cooperation and creativity, including the characteristics of biological demographic characteristics, the relationship between human personality, gender, ability and creativity, as well as the specific network relationship. Research continues to focus on the relationship between subject characteristics and creativity, network relations, network structure, climate and creativity in knowledge networks.

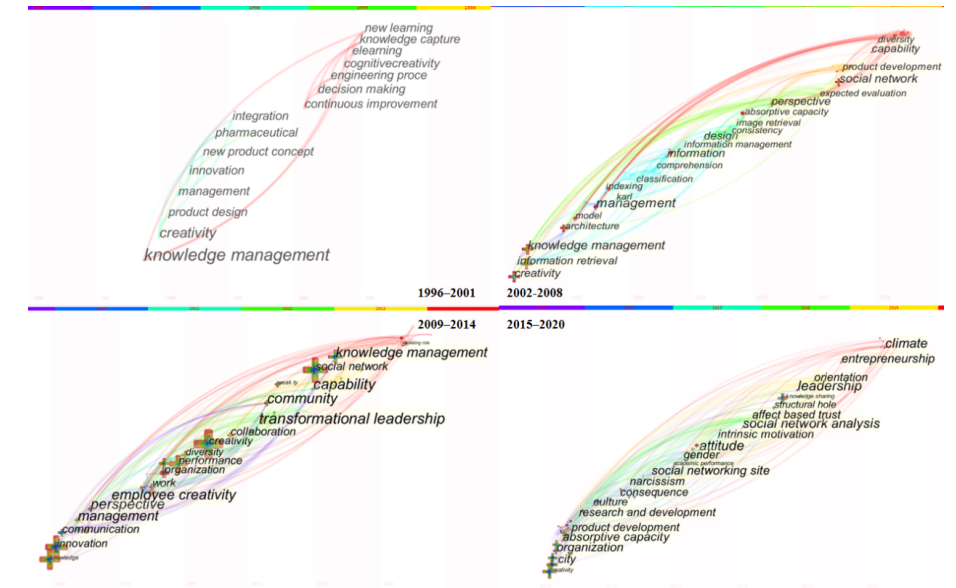

Figure 6. The time-zone visualization of knowledge networks and creativity research

\section{Conclusion and Future Research}

\subsection{Conclusion}

Although there has been extensive research on knowledge networks and creativity, including several qualitative reviews, a comprehensive bibliometric review was needed to visualize quantitatively the landscape and evolution of knowledge networks and creativity literature. To achieve this goal, this study uses CiteSpace to detect and investigate the knowledge networks and creativity articles published from 1996 to 2020. By analyzing the publication time, national/region, co-citations of authors, documents, and journals, the time-zone visualization of knowledge networks and creativity research published from WOS, the following conclusions can be drawn:

The number of published papers on knowledge networks and creativity has been on the rise year by year, especially since 2006, which has become and will continue to be the frontier hotspot of academic research. The United States is the most influential country in this field, followed by China, United Kingdom and other countries.

Through journal co-citation analysis, influential articles on knowledge networks and creativity are published in top-tier management journals. Knowledge networks and creativity is a popular topic attracting significant attention from researchers in previous three decades. It is the research hotspot and frontier of management.

By analyzing the co-citations of authors, documents, major researchers and topics in the field of knowledge networks and creativity are presented. The authors Perry-Smith JE, Amabile TM, Burt RS, Nonaka I, Hansen MT. play an important role in the evolution of knowledge networks and creativity research. These five articles are the most influential knowledge networks and creativity works: Perry-Smith JE (2006), Zhou J (2009), Fleming L (2007), Baer M (2010), Phelps C (2012). These five articles providing new and comprehensive insights into knowledge networks and creativity research and should be read by newcomers.

Through keyword time zone analysis, we can summarize the research evolution process of knowledge networks and creativity. The evolution process of hot spots is as follows: the relationship between knowledge and creativity, the relationship between social network knowledge and creativity, the structural characteristics of knowledge networks and creativity, and the subject of knowledge and creativity.

\subsection{Future Research}

Through literature review and combining with the latest research trends, this paper summarizes the future research content: 


\subsubsection{The Moderating Effect between Knowledge Networks Characteristics and Creativity}

Further research is necessary to identify moderating variables that affect the relationship between network structure characteristics and knowledge output. The study of moderating effects will help to understand how to design and manage knowledge networks that contribute to creativity and the related policy mechanisms.

\subsubsection{The Influence of the Formation and Dynamic Evolution of Knowledge Networks on Creativity}

Knowledge networks are in the dynamic evolution, the formation and change mechanism of network is a topic that cannot be ignored. For example, Perry-Smith JE and Shalley (Perry-Smith \& Shalley, 2014; Shalley, 2003) found that creativity helps to improve the network centrality and the number of strong ties of individuals. However, with the increase of centrality, the level of individual creativity improvement tends to weaken. When centrality reaches a certain level, it will limit creativity. Also, network structure (member centrality, structural hole, network ties, network size) and network quality (opportunistic behavior, reputation, trust) also affect the value of network to members and network sponsors (Afuah, 2013). When the attributes of subjects or the environment of social relations change, the knowledge networks will show dynamic changes. The research on the formation and dynamic evolution of knowledge networks will provide some managerial inspirations for creativity from the perspective of network management.

\subsubsection{The Multilevel Nesting Effect of Knowledge Networks}

The conclusions of cross-level research will contribute to a more comprehensive understanding of the relationship between knowledge networks and creativity. Through comprehensive and in-depth analysis of the influence of different characteristics of innovation systems on knowledge creation, comprehensive suggestions can be put forward for innovation policy and management.

\subsubsection{The Influence of Knowledge Networks on Radical Creativity and Incremental Creativity}

Incremental creativity is the improvement of existing knowledge, while radical creativity is the subversion of existing knowledge (Madjar, Greenberg, \& Chen, 2011). At present, some studies have proposed the influence of knowledge networks characteristics on these two types of creativity, but more empirical tests are still lacking. More theoretical analysis and empirical research need to be studied.

\section{References}

Afuah, A. (2013). Are network effects really all about size? The role of structure and conduct. Strategic Management Journal, 34(3), 257-273. https://doi.org/10.1002/smj.2013

Antonakis, J., Bastardoz, N., Jacquart, P., \& Shamir, B. (2016). Charisma: An ill-defined and ill-measured gift. Annual Review of Organizational Psychology and Organizational Behavior, 3(1), 293-319. https://doi.org/10.1146/annurev-orgpsych-041015-062305

Baer, M. (2010). The Strength-of-Weak-Ties Perspective on Creativity: A Comprehensive Examination and Extension. Journal of Applied Psychology, 95(3), 592-601. https:// doi.org/10.1037/a0018761

Baer, M. (2012). Putting Creativity to Work: The Implementation of Creative Ideas in Organizations. Academy of Management Journal, 55(5), 1102-1119. https://doi.org/10.5465/amj.2009.0470

Baggio, R., \& Valeri, M. (2020), Network science and sustainable performance of family businesses in tourism, Journal of Family Business Management. https://doi.org/10.1108/JFBM-06-2020-0048

Bavelas, \& Alex. (1950). Communication Patterns in Task-Oriented Groups. The Journal of the Acoustical Society of America, 22(6), 725-730. https://doi.org/10.1121/1.1906679

Burt, E. L., \& Atkinson, J. (2012). The relationship between quilting and wellbeing. Journal of Public Health, 34(1), 54-59. https://doi.org/10.1093/pubmed/fdr041

Chen, C. (2013). Mapping Scientific Frontiers. London: Springer.

Chen, C. (2006). CiteSpace II: Detecting and visualizing emerging trends and transient patterns in scientific literature. Journal of the American Society for Information Science and Technology, 57(3), 359-377. https://doi.org/10.1002/asi.20317

Chen, Y., Chen, C., Liu, Z., Hu, Z., \& Wang, X. (2015). Methodological function of CiteSpace knowledge graph. Studies in Science of Science, 33(2), 242-253.

Chen, Y., Liu, Z., Chen, J., \& Hou, J. (2008). Development process of scientific knowledge graph. Studies in Science of Science, (3), 449-460.

Liu, S. and Chen, C. (2012). The proximity of co-citation. Scientometrics, 91(2), 495-511. 
https://doi.org/10.1007/s11192-011-0575-7

Madjar, N., Greenberg, E., \& Chen, Z. (2011). Factors for Radical Creativity, Incremental Creativity, and Routine, Noncreative Performance. Journal of Applied Psychology, 96(4), 730-743. https://doi.org/10.1037/a0022416

Perry-Smith, J. E. (2006). Social Yet Creative: The Role of Social Relationships in Facilitating Individual Creativity. Academy of Management journal, 49(1), 85-101. https://doi.org/10.5465/AMJ.2006.20785503

Perry-Smith, J. E., \& Shalley, C. E. (2014). A Social Composition View of Team Creativity: The Role of Member Nationality-Heterogeneous Ties Outside of the Team. Organization Science, 25(5), 1434-1452. https://doi.org/10.1287/orsc.2014.0912

Phelps, C., Heidl, R., \& Wadhwa, A. (2012). Knowledge, Networks, and Knowledge Networks: A Review and Research Agenda. Journal of management, 38(4), 1115-1166. https://doi.org/10.1177/0149206311432640

Shalley, J. E. P. A. (2003). The Social Side of Creativity: A Static and Dynamic Social Network Perspective. Academy of Management Review, 28(1). https://doi.org/10.5465/amr.2003.8925236

Tsai, W., \& Wu, C. H. (2010). Knowledge combination: A co-citation analysis. Academy of Management Journal, 53(3), 441-450. https://doi.org/10.5465/amj.2010.51459152

Valeri, M., \& Baggio, R. (2020a). Social network analysis: organizational implications in tourism management. International Journal of Organizational Analysis. https://doi.org/10.1108/IJOA-12-2019-1971

Valeri, M., \& Baggio, R. (2020b). Italian tourism intermediaries: a social network analysis exploration. Current Issues in Tourism. https://doi.org/10.1080/13683500.2020.1777950

Zhou, J., Shin, S. J., Brass, D. J., Choi, J., \& Zhang, Z. (2009). Social networks, personal values, and creativity: Evidence for curvilinear and interaction effects. Journal of Applied Psychology, 94(6), 1544-1552. https://doi.org/10.1037/a0016285

\section{Copyrights}

Copyright for this article is retained by the author(s), with first publication rights granted to the journal.

This is an open-access article distributed under the terms and conditions of the Creative Commons Attribution license (http://creativecommons.org/licenses/by/4.0/). 